\title{
Controlling quantum information processing in hybrid systems on chips
}

\author{
Guy Bensky • Robert Amsüss • Johannes Majer • \\ David Petrosyan · Jörg Schmiedmayer • \\ Gershon Kurizki
}

Received: 9 June 2011 / Accepted: 30 August 2011 / Published online: 4 October 2011 (C) The Author(s) 2011. This article is published with open access at Springerlink.com

\begin{abstract}
We investigate quantum information processing, transfer and storage in hybrid systems comprised of diverse blocks integrated on chips. Strong coupling between superconducting (SC) qubits and ensembles of ultracold atoms or NV-center spins is mediated by a microwave transmission-line resonator that interacts near-resonantly with the atoms or spins. Such hybrid devices allow us to benefit from the advantages of each block and compensate for their disadvantages. Specifically, the SC qubits can rapidly implement quantum logic gates, but are "noisy" (prone to decoherence), while collective states of the atomic or spin ensemble are "quiet"(protected from decoherence) and thus can be employed for storage of quantum information. To improve the overall performance (fidelity) of such devices we discuss dynamical control to optimize quantum state-transfer from a "noisy" qubit to the "quiet" storage ensemble. We propose to maximize the fidelity of transfer and storage in a spectrally inhomogeneous spin ensemble, by pre-selecting the optimal spectral portion of the ensemble. Significant improvements of the overall fidelity of hybrid devices are expected under realistic conditions. Experimental progress towards the realization of these schemes is discussed.
\end{abstract}

Keywords Quantum information · Hybrid quantum systems · Atom chip

\footnotetext{
G. Bensky · G. Kurizki

Department of Chemical Physics, Weizmann Institute of Science, 76100 Rehovot, Israel

R. Amsüss · J. Majer · J. Schmiedmayer $(\varangle)$

Vienna Center for Quantum Science and Technology (VCQ), Atominstitut, TU-Wien, Stadionalle 2, 1020 Vienna, Austria

e-mail: Schmiedmayer@atomchip.org

D. Petrosyan

Institute of Electronic Structure and Laser, FORTH, 71110 Heraklion, Crete, Greece
} 


\section{Introduction}

Each quantum system considered for quantum information processing has its strengths and weaknesses. Some systems are better suited for processing, others for storage, and there are those best suited for writing-in/reading-out or transferring quantum information. Realistically, to build robust quantum information systems, one will need to combine different systems to pool their strengths in order to overcome their weaknesses. This has prompted the suggestion of hybrid, composite quantum systems [19] that would combine the advantages of their different subsystems. An important requirement of such hybrids is to faithfully transfer qubits between the subsystems.

Such hybrid quantum devices may be envisioned to integrate very different subsystems, or "blocks" on a single chip. As a general example, but without loss of generality, we will discuss here systems that are constructed around superconducting qubits. On a superconducting chip we envision three main components: (1) A quantum-processor block, (2) a quantum memory block and (3) a quantum "bus" that acts as an interface:

- The envisioned quantum-processor is based on superconducting qubits [10-12] that can rapidly and efficiently perform quantum gate operations. Such qubits are vulnerable to decoherence due to their strong coupling to the environment and the noise of the external controls.

For the purpose of this paper we can view the superconducting qubit as an artificial atom with a strong anharmonicity and a very strong coupling to the electromagnetic field [13]. The anharmonicity of the potential defining the qubit states ensures that one creates in a single qubit only a single excitation like in a single atom. The strong coupling to the electromagnetic field allows efficient coupling to the quantum "bus" discussed below

- The quantum-memory block is weakly coupled to the environment and the external controls, and is robust against decoherence and therefore suitable for implementing a long lived storage of quantum information. This may be a single isolated system like a trapped ion [14], or a collective qubit encoded in, for example, a spin ensemble [15].

For the purpose of this paper we concentrate on collective states encoded in spin ensembles as the memory qubit. These can be very robust and can have coherence properties similar to a single spin $[16,17]$. The two systems we consider here are (1) an atomic physics implementation where the qubit is encoded in the hyperfine ground states of (trapped) atoms or molecules [9,18-20]; (2) a solid state implementation where the qubit is encoded in the electron or nuclear spin states of nitrogen-vacancy (NV) color centers in diamond [21-24]

- The interface or quantum "bus", mediates among the different blocks or qubits and allows therefore coupling to the quantum memory. It should be designed to provide strong coupling and high-fidelity transfer of qubits to the quantum memory.

Here we consider as the bus a transmission line microwave cavity [25]. Such cavities have very small mode volume (typically $\ll 10^{-4} \lambda^{3}$ ), which results in a large field per photon and consequently in a strong electromagentic interaction allowing to wire up quantum systems [4]. In addition, the interaction with the different qubits can quickly switched on and off by, e.g., tuning them in and out of resonance. 
Even though the mode volume of chip-based microwave cavities is very small, their coupling to the long lived spin states is very weak (typically $100 \mathrm{~Hz}$ ). This limitation can be overcome by using polar molecules [1-3] or Rydberg atoms [5,6,26,27] which couple strongly to the cavity field to assist the transfer of quantum information (QI). Alternatively, we may employ ensembles with $N \gg 1$ spins (atoms), whose $\sqrt{N}$ scaling enhances the coupling to collective states of the ensemble [8,9,28]. Recent experimental demonstrations of trapping atoms on superconducting atom chips [2934], decelerating and trapping of polar molecules on a chip [35], and strong coupling between spin ensembles and microwave photons in superconducting cavities [36-40] are important steps towards implementing functional, hybrid quantum devices of this kind.

Implementations using trapped atoms or molecules on a chip [41] have the advantage that the ensembles are homogeneous, but small and require the large overhead of cooling and trapping to be implemented in a cryogenic environment at $\mathrm{mK}$ temperatures. On the other hand, implementations using solid-state based spin ensembles are simpler to prepare and handle at $\mathrm{mK}$ temperatures, and will allow large ensembles with $N \sim 10^{12}$ but are prone to inhomogeneous spectral broadening.

This broadening can both reduce the fidelity of the QI transfer and limit its coherent storage time [42-44]. Although spin-echo methods [45] can in principle prolong the storage time, they are restricted by the requirement to be faster than the inverse spectral width of the spin ensemble $[46,47]$. In addition, these methods cannot compensate for the fidelity loss during the QI transfer/retrieval.

In this work we discuss possible avenues for realizing such integrated hybrid quantum devices. QI transfer between the cavity and the ensemble-based quantum memory is typically the main experimental bottleneck. The coupling between the processing (superconducting) qubit and the cavity is, by contrast, very strong and can be quickly switched on and off at will [25,48-51].

It is interesting to note that at present, high-Q cavities are the best media for quantum-state storage (see, for example, Ref. [52]). Hence, the quality benchmark of integrated hybrid devices should be such that as to allow longer or higher-fidelity storage than such cavities. We discuss the prospects for achieving this benchmark.

The paper is organized as follows: We first give an overview of the superconducting qubit-cavity coupling, Sect. 2, and then concentrate on the coupling and transfer to the quantum memory, Sects. 3-6. We discuss how the fidelity of this transfer, and of the entire hybrid QI device, can be significantly improved by combining several methods of dynamical control: (i) In Sect. 3 we examine a strategy for maximizing the average fidelity of quantum state-transfer in such hybrids, from a noisy subspace (fragile under decoherence), used for QI writing / reading, to a quiet (robust) subspace, used for memory / storage, by choosing an appropriate dynamical control field. (ii) In Sect. 4 we analyze the effect 'of optimal spectral filtering of the inhomogeneous atomic or spin ensemble on the total fidelity of transfer, memory and retrieval. In Sects. 5 and 6 we then discuss these strategies in the context of three different implementations, and point out in Sect. 7 ways to achieve the quality benchmark discussed above. 


\section{Superconducting qubit-cavity coupling}

To have in mind a concrete scenario, we consider a solid state qubit that is represented by a SC Cooper pair box coupled to a SC electrode via two tunnel junctions at the rate $E_{J}$ (charge qubit) in the SQUID configuration [12]. At the charge degeneracy point, the energy separation $\hbar \omega_{10}=2 E_{J} \cos \left(\pi \Phi / \Phi_{0}\right)$ between the qubit states $|0\rangle$ and $|1\rangle$ can be dynamically controlled via an external magnetic field $B_{\perp}$ that induces flux $\Phi=A B_{\perp}$ through the SQUID area $A\left(\Phi_{0}=h c / 2 e\right.$ is the flux quantum). Typical values for $E_{J} / \hbar$ are the microwave range (10-20 GHz). The dipole moment $\wp_{01}$ for the transition $|0\rangle \leftrightarrow|1\rangle$ is typically very large, $\wp_{01} \simeq 10^{4} a_{0} e$. Thus, many fast quantum logic gates can be implemented within the qubit dephasing time $1 / \gamma_{q} \gtrsim 1 \mu \mathrm{s}$ [53].

Charge qubits can be embedded in near-resonant SC transmission line resonators, such as a coplanar waveguide (CPW) cavity of Fig. 1a [4,25,48-50], having a high quality factor $Q$ up to $10^{6}$. The tight confinement of the cavity field in a small volume (see below) yields very large field per photon $\varepsilon_{c}$ and strong coupling (vacuum Rabi frequency) $\eta_{q c}=\left(\wp_{01} / \hbar\right) \varepsilon_{c} u(\mathbf{r}) \sim 2 \pi \times 50 \mathrm{MHz}$ between the cavity field and the qubit located at position $\mathbf{r}$ near the field antinode where the cavity mode function $u(\mathbf{r}) \lesssim 1$. In the frame rotating with the cavity field frequency $\omega_{c}$, the Hamiltonian has the form

$$
H_{q c}=\hbar \delta_{q c} \hat{\sigma}^{+} \hat{\sigma}^{-}-\hbar \eta_{q c}\left(\hat{\sigma}^{+} \hat{c}+\hat{c}^{\dagger} \hat{\sigma}^{-}\right)
$$

where $\delta_{q c}=\omega_{10}-\omega_{c}$ is the externally controlled (via $\left.B_{\perp}\right)$ detuning, $\hat{\sigma}^{-}\left(\hat{\sigma}^{+}\right)$is the qubit lowering (rising) operator, and $\hat{c}\left(\hat{c}^{\dagger}\right)$ is the cavity photon annihilation (creation) operator.

One can incorporate many SC qubits in the same CPW cavity, each qubit located near the cavity field antinode (Fig. 1a). The cavity can then mediate long-range controlled interactions between pairs of resonant qubits [25,48-50,4], realizing, e.g., the two-qubit $\sqrt{\mathrm{SWAP}}$ gate, which together with the single qubit rotations form the universal set of logic gates in such a quantum computer. However, due to rapid dephasing and relaxation, neither SC qubits nor the cavity mode can carry out reliable long term storage of QI. In what follows, we show that this task can be accomplished by dynamical control of the QI transfer to the quantum memory ensemble positioned

(a)

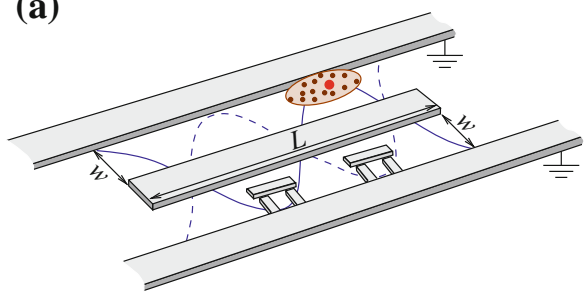

(b)

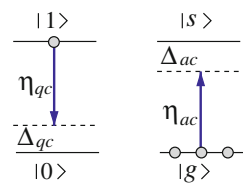

Fig. 1 a CPW cavity with strip-line length $L$ and electrode distance $w$. SC qubits are placed at the antinodes of the standing wave field and ensembles of ultracold atoms or NV centers are placed near the CPW surface. b SC qubit (left) and ensemble qubit (right) can couple to a common mode of the CPW cavity 
near or at the surface of an integrated chip that incorporates the CPW cavity and SC qubits.

As we will show in Sect. 6, to achieve high fidelity gates using CPW interface it is crucial to suppress the cavity temperature corresponding to less than 0.01 thermal photons.

\section{Optimized transfer by dynamical control}

We use dynamical control to optimize a reliable transfer of a quantum state from the fragile (noisy) SC qubit to the robust (quiet) memory qubit via the cavity. We choose to focus on the case of two resonant qubits with temporally controlled coupling strength as an example, since it is simple enough to be solvable analytically yet still holds surprising results. However, the same method can be numerically applied to more complex systems. Our goal here is to devise the best solution when the transfer is limited by the homogeneous broadening (decoherence) of the SC qubit and the cavity, rather than by the inhomogeneous broadening of the memory (to be discussed in Sect. 4).

The free Hamiltonian of a system of two qubits with controlled coupling is

$$
\begin{aligned}
\hat{H}(t) & =\hat{H}_{S}(t)+\hat{H}_{I}+\hat{H}_{B} \\
\hat{H}_{S}(t) & =\frac{\omega_{0}}{2}\left(\hat{\sigma}_{z}^{(1)}+\hat{\sigma}_{z}^{(2)}\right)+H_{c}(t), \\
\hat{H}_{c}(t) & =V(t) \hat{\sigma}_{x}^{(1)} \otimes \hat{\sigma}_{x}^{(2)} .
\end{aligned}
$$

Here $H_{B}$ is the Hamiltonian for the environment (bath), $H_{I}$ is the coupling operator between the system and the environment and $\hat{H}_{c}(t)$ is the Hamiltonian (or effective Hamiltonian in some cases) for the controlled interaction between the qubits, $V(t)$, describing the adjustable amplitude of the interaction (see Fig. 2 for examples of how such adjustable coupling amplitudes can be achieved in practice). Since our goal is to study a system with decoherence only in the noisy qubit 1 , the system-bath interaction Hamiltonian $H_{I}$ is taken to be

$$
\hat{H}_{I}=\hat{S} \otimes \hat{B}=\hat{\sigma}_{z}^{(1)} \otimes \hat{B}
$$

where $\hat{B}$ is a bath operator. There is no restriction on the choice of either $B$ or $H_{B}$. This model represents a single noise source of proper dephasing in the source qubit 1 due to the bath operator $\hat{B}$, whereas the target qubit 2 is robust against decoherence. This model can be generalized to any degree of asymmetry between the decoherence properties of the two qubits.

The accumulated phase

$$
\phi(t)=\int_{0}^{t} V\left(t^{\prime}\right) d t^{\prime}
$$


(a)

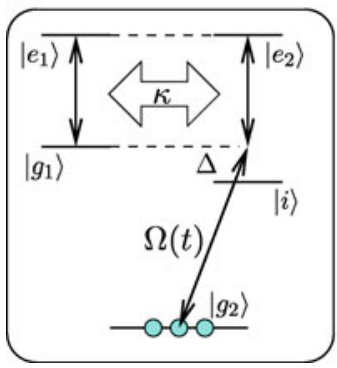

(b)

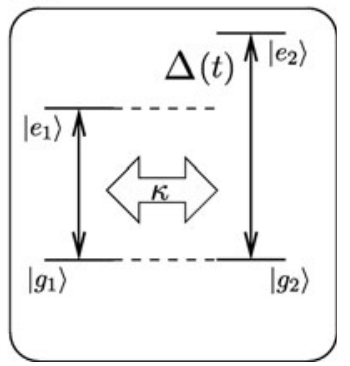

Fig. 2 Schemes of dynamically controllable coupling between "noisy" source qubit 1 and quiet target qubit 2: a 2-photon transfer off-resonantly through $|i\rangle$ gives effective $\sigma_{x}^{1} \otimes \sigma_{x}^{2}$ coupling with a controllable strength $V(t)=\frac{\kappa \Omega(t)}{\Delta}$, where $\Omega(t)$ is the Rabi frequency of an external laser field. This allows for a smooth control, but limits the coupling to $V(t) \lesssim \kappa / 10$. b Controlled detuning of the source qubit from the target qubit $\Delta(t)$. This is limited to an "on/off" control, but allows a coupling of up to $V(t)=\kappa$, where $\kappa$ is the natural (non-controlled) coupling between the qubits

is our control function. In the ideal case, without decoherence or leakage, the state transfer from qubit 1 to qubit 2 can be perfectly realized if at the final time, $t_{f}$, the phase $\phi(t)$ satisfies $\phi\left(t_{f}\right)=\frac{\pi}{2}$, whence any initial state of qubit 1 is mapped onto that of qubit 2 (initially in the ground state)

$$
\left(\alpha\left|g_{1}\right\rangle+\beta\left|e_{1}\right\rangle\right)\left|g_{2}\right\rangle \rightarrow\left|g_{1}\right\rangle\left(\alpha\left|g_{2}\right\rangle-i \beta\left|e_{2}\right\rangle\right)
$$

for any normalized $\alpha, \beta$. Here the states $\left|g_{1}\right\rangle\left(\left|g_{2}\right\rangle\right)$ and $\left|e_{1}\right\rangle\left(\left|e_{2}\right\rangle\right)$ are respectively the ground and the excited states of the source qubit 1 and the target qubit 2.

There are two conflicting noise (error) considerations for the transfer, each affecting a different subsystem: (i) In the presence of interaction between the source qubit 1 and the bath, the longer the information stays in qubit 1 the lower the fidelity of the transfer. (ii) On the other hand, if we make the transfer extremely fast, it may result in population from $\left|g_{1}\right\rangle\left|g_{2}\right\rangle$ leaking into $\left|e_{1}\right\rangle\left|e_{2}\right\rangle$, thus lowering the fidelity of transfer. Such leakage [54-60] signifies the violation of the rotating wave approximation (RWA). Namely, fast modulation $V(t)$ may incur unwanted, off-resonant, transitions if the transfer rate is comparable to the energy difference (level distance) of the qubits, $\omega_{0}$.

We here focus on bath-related errors (i), assuming that the RWA is valid, i.e., there is no leakage because of the RWA violation. This may be the case if the transfer time is much slower than the energy separation $\omega_{0}$. The control Hamiltonian $H_{c}(t)$ then has the RWA form [61-65]:

$$
\hat{H}_{c}(t) \equiv V(t) H_{c} ; \quad H_{c}=\left|e_{1} g_{2}\right\rangle\left\langle g_{1} e_{2}|+| g_{1} e_{2}\right\rangle\left\langle e_{1} g_{2}\right|
$$

The average fidelity of the transfer has an involved dependence on the modulation $V(t)$ and the transfer time $t_{f}$. The problem at hand is to find the optimal transfer that 
minimizes the average error at time $t_{f}, 1-\overline{\mathcal{F}\left(t_{f}\right)}, \overline{\mathcal{F}}$ being the fidelity averaged over all possible initial states of qubit 1 , with equal distribution over the Bloch sphere.

Obviously, zero error is obtainable for infinitely fast (zero-time) transfer, if we allow infinitely strong control field. Since this is unphysical, we add a constraint on the total energy $E$ of the field that causes the transfer process

$$
\int_{0}^{t_{f}} d t(V(t))^{2}=\int_{0}^{t_{f}} d t\left(\frac{d \phi(t)}{d t}\right)^{2}=E .
$$

Although other constraints may be considered, this constraint is both simple and physical: if we use the scheme described in Fig. 2a the energy constraint translates to a limit on the average laser intensity. In addition, using this constraint can prevent leakage to levels out of the operational qubit subspace [54-60]. The constraint defines the minimum possible time for the transfer $t_{\min }=\frac{\pi^{2}}{4 E}$. Choosing an exact value of the energy might seem arbitrary since one usually has some freedom as to the amount of energy the system can handle. However, the exact value we choose would only matter if a small change in the energy could result in a large change in the fidelity. Yet, we have shown numerically and analytically that the error and optimal modulation change smoothly with the energy constraint and are nearly linear in the characteristic transfer time $E^{-1}$. Hence, the chosen energy constraint is simply a typical value allowed for the process.

Consider in what follows a typical non-Markovian Lorentzian bath spectrum, i.e., the correlation function $\Phi(t)$ of the bath operator $B$ is exponentially decaying $\Phi(t)=\frac{\gamma}{t_{c}} e^{-|t| / t_{c}}, t_{c}$ being the correlation (memory) time. One might expect that for such a simple bath the best strategy is the fastest possible transfer under the energy constraint, i.e. when the modulation is given by $V\left(0 \leq t \leq t_{\min }\right)=2 E / \pi$. Surprisingly, a slower transfer $\left(t_{f}>t_{\min }\right)$ under an appropriate modulation $\phi(t)$ (detailed below) can improve the average fidelity even for a purely Markovian bath, with negligible correlation (memory) time $t_{c} / t_{\min } \rightarrow 0$, and more so for baths with memory times longer than the transfer time, $t_{c} \gtrsim t_{\min }$.

a. Markovian limit - When the bath is memoryless, i.e., Markovian, this improvement is limited, as shown in Fig. 4, to about 12\%. By comparing the "best" solution to the "fastest" one (Fig. 3), one can see the the "best" solution starts off faster and then slows down, being overtaken by the "fastest" solution only at $t \approx 0.9 t_{\min }$. This illustrates what we believe to be the source of the Markovian-noise error reduction: the "best" solution starts off faster, so as to transfer more of the information while it is still nearly untainted by the bath. Obviously, towards the end it must slow down so as to comply with the energy constraint, thus resulting in total transfer time $t_{f}$ that is longer than the fastest time $t_{\min }$ for the given energy.

This system is analytically solvable in the Markovian limit, under the assumption that the effect of the bath is weak during the entire transfer. In the frame rotating with $H_{S}(t)$, and using the RWA from Eq. (5), the Lindblad master equation of the system is [66] 
(a) Markovian bath: $t_{c} / t_{\min }=0$

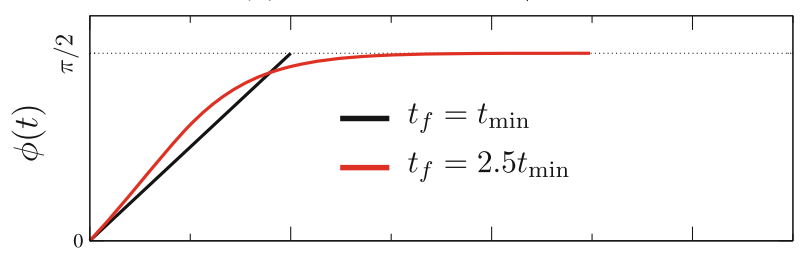

(b) Non-Markovian bath: $t_{c} / t_{\min }=10$

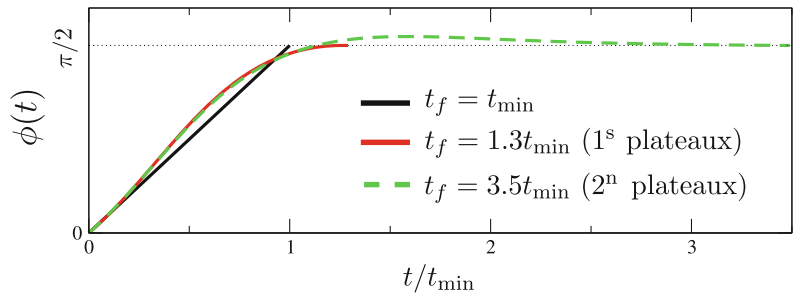

Fig. 3 The transfer phase $\phi(t)$ versus $t / t_{\min }$. The fastest modulation (black, dotted) with the Markovian optimal modulation (red, solid) and the non-Markovian optimal modulation (green, dashed), in Markovian (a) and non-Markovian (b) baths. In the Markovian bath the optimal modulation transfer starts off faster than the "fastest" transfer (when the information is still "fresh"), and slows down subsequently. For the non-Markovian bath, optimal modulation achieves full transfer $(\phi(t)=\pi / 2)$ well within the modulation, but then "overshoots" $(\phi(t)>\pi / 2)$, and eventually returns to $\phi(t)=\pi / 2$ (Color figure online)

$$
\begin{aligned}
\dot{\rho}(t)= & \gamma[2 S(t) \rho(t) S(t)-S(t) S(t) \rho(t)-\rho(t) S(t) S(t)] \\
S(t)= & e^{\imath H_{c} \phi(t)}\left|e_{1} g_{2}\right\rangle\left\langle e_{1} g_{2}\right| e^{-\imath H_{c} \phi(t)} \\
& {\left[e^{\imath H_{c} \phi(t)}\left|e_{1} g_{2}\right\rangle=\cos \phi\left|e_{1} g_{2}\right\rangle+\imath \sin \phi\left|g_{1} e_{2}\right\rangle\right] }
\end{aligned}
$$

where $\phi(t)=\int_{0}^{t} V\left(t^{\prime}\right) d t^{\prime}$ is the accumulated action on the system and $\gamma=\operatorname{Tr}\left\langle B B \rho_{B}\right\rangle$ is the Markovian decoherence rate.

We assume that the effect of the bath is weak and that in the frame rotating with $H_{S}(t)$ the density matrix $\rho(t)$ does not change much during the transfer $\rho(t) \approx \rho_{0}$. We can then find an expression for the fidelity of the system $f(t)$ at time $t$ given an initial state of $\left|\psi_{0}\right\rangle=\alpha\left|e_{1} g_{2}\right\rangle+\beta\left|g_{1} g_{2}\right\rangle,\left(\rho_{0}=\left|\psi_{0}\right\rangle\left\langle\psi_{0}\right|\right)$ :

$$
\begin{aligned}
\rho(t)= & \rho_{0}+\int_{0}^{t} \dot{\rho}\left(t^{\prime}\right) d t^{\prime} \\
f(t)= & \left\langle\psi_{0}|\rho(t)| \psi_{0}\right\rangle \\
\approx & \left\langle\psi_{0}\left|\rho_{0}\right| \psi_{0}\right\rangle \\
& +\gamma \int_{0}^{t} d t^{\prime}\left\langle\psi_{0}\left|2 S\left(t^{\prime}\right) \rho_{0} S\left(t^{\prime}\right)\right| \psi_{0}\right\rangle
\end{aligned}
$$




$$
\begin{gathered}
-\gamma \int_{0}^{t} d t^{\prime}\left\langle\psi_{0}\left|S\left(t^{\prime}\right) S\left(t^{\prime}\right) \rho_{0}+\rho_{0} S\left(t^{\prime}\right) S\left(t^{\prime}\right)\right| \psi_{0}\right\rangle \\
=1+2 \gamma \int_{0}^{t} d t^{\prime}\left(|\alpha|^{4} \cos ^{4} \phi\left(t^{\prime}\right)-|\alpha|^{2} \cos ^{2} \phi\left(t^{\prime}\right)\right)
\end{gathered}
$$

This is the fidelity for a specific initial state $\alpha|e\rangle+\beta|g\rangle$. By averaging this result over the entire Bloch sphere (all initial states) we find the average fidelity:

$$
\begin{aligned}
\overline{f(t)} & \approx 1+2 \gamma \int_{0}^{t} d t^{\prime}\left(\overline{|\alpha|^{4}} \cos ^{4} \phi\left(t^{\prime}\right)-\overline{|\alpha|^{2}} \cos ^{2} \phi\left(t^{\prime}\right)\right) \\
& =1+2 \gamma \int_{0}^{t} d t^{\prime}\left(\frac{1}{3} \cos ^{4} \phi\left(t^{\prime}\right)-\frac{1}{2} \cos ^{2} \phi\left(t^{\prime}\right)\right) \\
& =1-\gamma \int_{0}^{t} d t^{\prime}\left(\frac{1}{3} \cos ^{4} \phi\left(t^{\prime}\right)+\frac{1}{4} \sin ^{2} 2 \phi\left(t^{\prime}\right)\right)
\end{aligned}
$$

We can use the Euler-Lagrange variational method to find an analytical solution for the optimal modulation phase $\phi(t)$, given a Markovian $\left(t_{c} \rightarrow 0\right)$ bath, at long transfer times $t_{f} \rightarrow \infty$. The energy constraint gives

$$
E=\int_{0}^{\infty}(\dot{\phi}(t))^{2} d t \Rightarrow \frac{\partial E}{\partial \phi(t)}=-2 \ddot{\phi}(t) .
$$

The error $\left(1-\overline{f\left(t_{f}\right)}\right)$ we wish to minimize can be expressed, for $t_{f} \rightarrow \infty$, as:

$$
\begin{aligned}
\text { error } & \approx \frac{\gamma}{2} \int_{0}^{\infty}\left(\frac{\sin ^{2}(2 \phi(t))}{2}+2 \frac{\cos ^{4}(\phi(t))}{3}\right) d t \\
\Rightarrow \frac{\partial(\text { error })}{\partial \phi(t)} & \approx \frac{\gamma}{2}\left(\sin (4 \phi(t))+8 \frac{\cos ^{3}(\phi(t)) \sin (\phi(t))}{3}\right)
\end{aligned}
$$

With the Euler-Lagrange equation (using $-2 \lambda / \gamma$ as Lagrange multiplier), we obtain

$$
\begin{aligned}
\frac{\partial E}{\partial \phi(t)} & =-\frac{2 \lambda}{\gamma} \frac{\partial(\text { error })}{\partial \phi(t)} \\
\Rightarrow \frac{\partial E}{\partial \phi(t)} \dot{\phi}(t) & =-\frac{2 \lambda}{\gamma} \frac{\partial(\text { error })}{\partial \phi(t)} \dot{\phi}(t) \\
\frac{d}{d t}(\dot{\phi}(t))^{2} & =\lambda \frac{d}{d t}\left(\frac{\sin ^{2}(2 \phi(t))}{2}+2 \frac{\cos ^{4}(\phi(t))}{3}\right)
\end{aligned}
$$




$$
\begin{aligned}
(\dot{\phi}(t))^{2} & =\lambda\left(\frac{\sin ^{2}(2 \phi(t))}{2}+2 \frac{\cos ^{4}(\phi(t))}{3}\right) \\
\phi(t) & =\sqrt{\lambda} \int_{0}^{t} d t^{\prime} \sqrt{\frac{\sin ^{2}\left(2 \phi\left(t^{\prime}\right)\right)}{2}+2 \frac{\cos ^{4}\left(\phi\left(t^{\prime}\right)\right)}{3}}
\end{aligned}
$$

where we used the condition that $\phi(t) \underset{t \rightarrow \infty}{\longrightarrow} \pi / 2$ and $\dot{\phi}(t) \underset{t \rightarrow \infty}{\longrightarrow} 0$ to find the integration constant.

For $\lambda=1$, the Markovian phase $\phi_{M}(x)$ as a function of the dimensionless argument $x$ satisfies

$$
\frac{d \phi_{M}(x)}{d x}=\sqrt{\frac{\sin ^{2}\left(2 \phi_{M}(x)\right)}{2}+2 \frac{\cos ^{4}\left(\phi_{M}(x)\right)}{3}},
$$

with $\phi_{M}(0)=0$. Equation (13) can be solved numerically to determine the shape of $\phi_{M}(x)$ and its energy $e_{M}=\int_{0}^{\infty}\left|\phi_{M}^{\prime}(x)\right|^{2} d x=1.038 \ldots$ (where both $x$ and $e_{M}$ are dimensionless). By rescalling $\phi_{M}(x)$ we find the general Markovian optimal modulation at infitite time for any energy $E, \phi(t)=\phi_{M}\left(\frac{E}{e_{M}} t\right)$, with an error (1-fidelity) of $\frac{\gamma}{E} e_{M}^{2}=\frac{\gamma}{E} 1.077 \ldots, \gamma$ being the dephasing rate of the source qubit 1 . The fastest modulation with energy $E$ has an error of $\frac{\gamma}{E} \frac{\pi^{2}}{8}=\frac{\gamma}{E} 1.233 \ldots$ This means that the optimal modulation has about $12 \%$ less error than the fastest modulation for the same energy.

This improvement is not large: using this optimal solution will increase the fidelity from, e.g., 0.9 to 0.912 . Yet this is for a Markovian, memoryless, bath, where using current dynamical decoupling methods [56-60,67-71] no improvement was possible. For non-Markovian baths that cause the noisy-qubit decoherence the decrease in error (and hence increase in fidelity) can be much more significant, as shown below.

b. Non-Markovian bath effects - When the memory-time $t_{c}$ of the bath is comparable to or larger than the characteristic transfer time $t_{c} \gtrsim t_{\min }$, a much larger improvement can be achieved (see Fig. 4). Remarkably, the best solution actually performs a full transfer, $\phi(t)=\pi / 2$, well within the modulation time, but rather than stopping at $\phi=\pi / 2$ it then "overshoots" the transfer, so that $\phi(t)>\pi / 2$, and then returns slowly to $\pi / 2$ (see Fig. 3). This can explain the source of the noise reduction-when "overshooting", the information partially returns from the target (storage) qubit to the source (noisy) qubit, but with a negative sign. Hence, similarly to the "echo" method, the noise now operates in the reverse direction, correcting itself, i.e., the nonMarkovian bath effect is undone. This requires transfer times significantly larger than the minimal transfer time $t_{\mathrm{min}}$, ranging from $3 t_{\min }$ to $10 t_{\min }$ or more, yet the fidelity increases substantially (up to $50 \%$ in Fig. 4).

Two kinds of non-Markovian baths may play a role in the noisy SC qubit decoherence: (a) noisy controls with slow drift (memory) time [61-65]; (b) qubit relaxation in a cavity whose memory time is determined by its Q-factor [72]. 


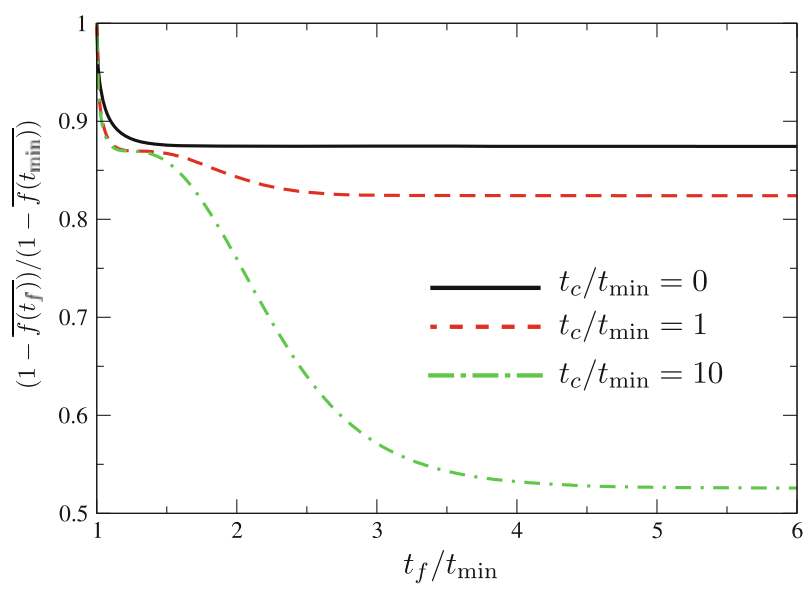

Fig. 4 Dependence of the lowest achievable average error $(\overline{1-\text { fidelity }})$ (averaged over all possible initial states of the source qubit) on the transfer time $t_{f}$ normalized to the error of the fastest transfer (over time $\left.t_{\min }\right)$ at a given transfer energy (Eq. 6). This function is plotted for various bath memory times: (black, solid) $t_{c}=0$ (Markovian); (red, dash) $t_{c}=t_{\min }$; (green, dash-dot) $t_{c}=10 t_{\min }$. Even for Markovian baths $\left(t_{c} \ll t_{\min }\right)$ the best solution is not the fastest one. For non-Markovian baths $\left(t_{c} \gtrsim t_{\min }\right)$ two plateaux (regions of insensitivity to $t_{f}$ ) can be seen. The first plateau is independent of the memory time, and matches the Markovian plateau. The second plateau is lower the longer the memory of the bath (Color figure online)

\section{Optimizing storage and transfer fidelities by spectral filtering of inhomogeneous spin ensembles}

When the coupling of a single spin or atom to the cavity field is weak, we have to encode a qubit in collective states of an ensemble of spins whose ground state is $\left|\psi_{0}\right\rangle=$ $\left|g_{1}, g_{2}, \ldots, g_{N}\right\rangle$ and the fully symmetrized, collective, single-excitation (Dicke) state is $\left|\psi_{1}\right\rangle=N^{-1 / 2} \sum_{j}|j\rangle$, where $|j\rangle=\left|g_{1}, g_{2}, \ldots, e_{j}, \ldots, g_{N}\right\rangle$ denotes a state with only spin $j$ excited. If all the spins had the same resonant frequency $\omega_{0}$, the singleexcitation state would evolve at time $t=\tau>0$ to $\left|\psi_{1}(\tau)\right\rangle=N^{-1 / 2} \sum_{j} e^{-i \omega_{0} \tau}|j\rangle$ and coherence is preserved if the position of the spins stays fixed.

In general spins in a solid state environment have different resonant frequencies $\omega_{j}$ which results in inhomogeneous broadening. Then, even if the symmetric state $\left|\psi_{1}\right\rangle$ is prepared at $t=0$, it would evolve into $\left|\tilde{\psi}_{1}(\tau)\right\rangle=N^{-1 / 2} \sum_{j} e^{-i \omega_{j} \tau}|j\rangle$, while the ground (or vacuum) state $\left|\psi_{0}\right\rangle$ remains unchanged. We thus define the "storage fidelity" as the squared overlap at time $\tau$ of state $\left|\psi_{1}(\tau)\right\rangle$ with its inhomogeneouslybroadened counterpart:

$$
\mathcal{F}(\tau) \equiv\left|\left\langle\psi_{1}(\tau) \mid \tilde{\psi}_{1}(\tau)\right\rangle\right|^{2}=\left|\frac{1}{N} \int n(\omega) e^{-i\left(\omega-\omega_{0}\right) \tau} d \omega\right|^{2}
$$

where $n(\omega)$ is the spectral density normalized to the total number of atoms, $\int n(\omega) d \omega=$ $N$.

Our goal is to modify the spectrum $n(\omega)$ of the atomic (spin) ensemble so as to maximize the fidelity of transfer, storage and retrieval. To this end, we employ a third 


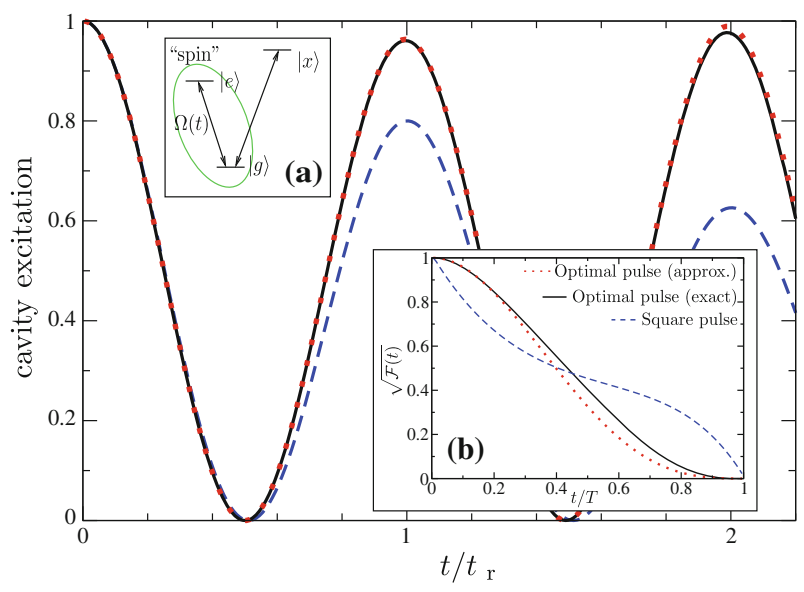

Fig. 5 Inset a atomic level scheme. Main panel: Rabi oscillations of a single excitation between the cavity and the atomic ensemble for the optimal pulse (exact solution: black solid line; approximate analytical solution: red dotted line), and the square pulse (blue dashed line). Inset $\mathbf{b}$ the corresponding $\sqrt{\mathcal{F}(t)}$ for preparation time $T=10 t_{\mathrm{tr}}$ (Color figure online)

(auxiliary), long-lived state $|x\rangle$, outside the spin- $\frac{1}{2}$ space of the ground $|g\rangle$ and excited $|e\rangle$ states used for QI storage (see Fig. 5a). The preparation of the ensemble proceeds in three steps: (1) Starting with the entire ensemble in the ground state $|g\rangle$, apply an external pulse of Rabi frequency $\Omega(t)$ exciting the atoms to the state $|e\rangle$. The duration $T$ of the pulse should be long enough in order to select only the atoms with transition frequencies $\omega$ within a desired range of $\delta \sim 2 \pi / T$ around $\omega_{0}$, while the shape of the pulse $\Omega(t)$ is designed to optimize the resulting frequency spectrum (see below). (2) Transfer all those atoms remaining in state $|g\rangle$ to the auxiliary, long lived state $|x\rangle$ by another strong pulse, using, e.g., an adiabatic sweep across the $|g\rangle \rightarrow|x\rangle$ transition $[73,74]$. (3) Return the atoms selected in stage (i) from $|e\rangle$ to $|g\rangle$ by, e.g., the adiabatic transfer.

The chosen subensemble is now ready to use. Its spectrum is $n(\omega) P\left(\omega-\omega_{0}\right)$, where

$$
P\left(\omega-\omega_{0}\right)=\left|\left\langle e\left|T_{+} e^{-i \int_{0}^{T} H(t) d t}\right| g\right\rangle\right|^{2},
$$

with $H(t)=\frac{1}{2}\left(\omega-\omega_{0}\right) \sigma_{z}+\Omega(t) \sigma_{x}$. Equation (15) is the probability for the preparation pulse with frequency $\omega_{0}$ and envelope $\Omega(t)$ in step 1) to excite an atom (spin) with resonant frequency $\omega$.

If $P(\omega)$ is much narrower than the initial spectrum of the atoms $n(\omega)$, we may replace $n(\omega) \rightarrow n\left(\omega_{0}\right)$, obtaining

$$
\mathcal{F}(\tau) \approx \frac{4 \pi^{2} n^{2}\left(\omega_{0}\right)}{N^{2}}\left|\int_{0}^{T} d t \Omega(t+\tau) \Omega(t)\right|^{2}
$$


where $N=2 \pi n\left(\omega_{0}\right) \int_{0}^{T} d t \Omega^{2}(t)$. Hence, for $\tau>T$ the fidelity vanishes and one cannot store the QI for a time longer than the preparation time.

To determine the optimal preparation pulse-shape $\Omega(t)$, we use the Euler-Lagrange method to maximize the fidelity (Eq. 16) for a specific storage time $\tau$, while fixing the number of atoms $N$ and the preparation time $T$.

For $\tau \ll T$ we find

$$
\Omega(t)=\Omega_{0} \sin (\pi t / T), \quad N=\pi n\left(\omega_{0}\right) \Omega_{0}^{2} T .
$$

The resulting storage fidelity of the atomic ensemble is given by

$$
\mathcal{F}(\tau)=\left|\frac{(T-\tau) \cos (\pi \tau / T)}{T}+\frac{\sin (\pi \tau / T)}{\pi}\right|^{2} \approx 1-\frac{\pi^{2} \tau^{2}}{T^{2}} .
$$

Hence, using the preparation pulse of Eq. (17), the fidelity loss for small times $\tau \ll T$ becomes quadratic in $\tau$, which should be contrasted with linear fidelity loss for the square preparation pulse (Fig. 5b). This can greatly improve the ensemble coherence time. We illustrate these results for NV-centers in Sect. 5.2

\section{Magnetic coupling of the cavity field to spin ensembles}

The magnetic dipole coupling to a microwave field is much weaker than the electric dipole coupling to optical-fields. Nevertheless one can achieve strong coupling to collective states of spin ensembles with currently available coplanar waveguide resonators (CPWR) due to their highly localized magnetic field. The high concentration of field energy near the surface results from a dramatic reduction of the effective volume $V_{\text {eff }} \sim \frac{\pi}{2} \lambda l^{2}$ of the CPWR mode, where $l$ is the decay length of the field which is of the order of the gap between the central conductor and the ground wire of the CPWR. For example, for the ${ }^{87} \mathrm{Rb}$ microwave transition at $6.83 \mathrm{GHz}$ (wavelength $\lambda \sim 3 \mathrm{~cm}$ ) and a typical decay length $l \sim 3 \mu \mathrm{m}$ one expects an enhancement of the atom-photon coupling strength of $(\lambda / l) \sim 10,000$. A full calculation of the local electromagnetic field [9] normalized to a single photon results in the field shown in Fig. 6b-d. We obtain at a distance of $1 \mu \mathrm{m}$ above the surface a field of $>40 \mu \mathrm{G}$ for a single photon, which confirms the simple estimate given above.

Below we will illustrate two different implementations of magnetic coupling to spin ensembles: (i) trapped ultracold atoms [9] and (ii) electron spins in diamond [36-38].

\subsection{Implementation in an atomic ensemble.}

Atom chips [75-77] are capable of trapping, positioning and manipulating large ensembles of ultracold atomic ensembles a few $\mu \mathrm{m}$ above a gap $[78,79]$. The advantage of atoms is that they do not have inhomogeneous broadening. Their disadvantage is that they are difficult to handle in a $\mathrm{mK}$ environment and cannot attain very large numbers $N$ : their $\sqrt{N}$ enhancement is currently limited to $\sim 10^{3}$. 


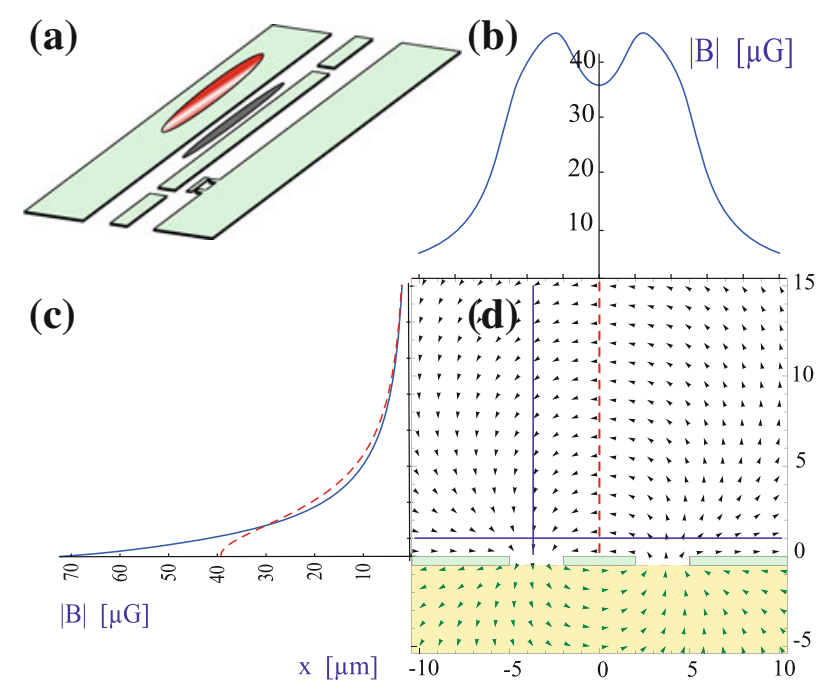

Fig. 6 a Schematic of the CPWR including a solid-state qubit and a cloud of ultracold atoms trapped above one of the gaps. $\mathbf{b}$ The magnetic field strength of a single photon as a function of lateral distance $1 \mu \mathrm{m}$ from the chip surface and $\mathbf{c}$ as a function of distance to the chip surface at the gap (full line) and at the central conductor (dashed line). d Vector plot of the magnetic field in the resonator

We envision [9] placing an ensemble of $10^{6}$ ultracold ${ }^{87} \mathrm{Rb}$ atoms with the ground state hyperfine splitting $\omega_{s g} / 2 \pi=6.83 \mathrm{GHz}$ between $|F=1\rangle \equiv|g\rangle$ and $\mid F=$ $2\rangle \equiv|s\rangle$ in the near field zone of the superconducting CPW cavity. Currently we are building up such an experiment $[80,81]$.

Let us choose the frequency of the CPW cavity to be near-resonant with that of the atomic transition $|g\rangle \leftrightarrow|s\rangle$ (Fig. 1b). When the atomic ensemble is near the field antinode, with the spacial dimension of the cloud being small compared to the mode wavelength, all the atoms couple symmetrically to the cavity field. The corresponding Hamiltonian can be expressed as

$$
H_{a c}=\hbar \delta_{a c} \hat{s}^{\dagger} \hat{s}+\hbar \eta_{a c}\left(\hat{s}^{\dagger} \hat{g} \hat{c}+\hat{c}^{\dagger} \hat{g}^{\dagger} \hat{s}\right)
$$

where $\delta_{a c}=\omega_{s g}-\omega_{c}$ is the detuning and $\eta_{a c}=i\left(\wp_{s g} / \hbar\right) \varepsilon_{c} u(\mathbf{r})$ is the coupling rate between the cavity field and a (single) atom at position $\mathbf{r}$. Since $|F=1\rangle \leftrightarrow|F=2\rangle$ is a magnetic dipole transition, the corresponding matrix element is small, $\wp_{s g} \simeq \frac{1}{2} \alpha a_{0} e$ with $\alpha=1 / 137$, which yields $\eta_{a c} \sim 2 \pi \times 40 \mathrm{~Hz}$ [for $u(\mathbf{r}) \lesssim 1$ ] [9]. The operators $\hat{g}\left(\hat{g}^{\dagger}\right)$ and $\hat{s}\left(\hat{s}^{\dagger}\right)$ annihilate (create) an atom in the corresponding state $|g\rangle$ and $|s\rangle$; these essentially bosonic operators live in a space of completely symmetrized states $\left|n_{g}, n_{s}\right\rangle$ with $n_{g}$ atoms in state $|g\rangle$ and $n_{s}$ atoms in state $|s\rangle$, while $n_{g}+n_{s}=N$.

With $10^{6}$ trapped ultracold ${ }^{87} \mathrm{Rb}$ atoms and a CPWR with a quality factor $Q \sim 10^{6}$ one can achieve strong coupling between a microwave photon in the CPWR and a collective hyperfine qubit state in the atomic ensemble with $g_{\text {eff }} / 2 \pi \sim 40 \mathrm{kHz}$ larger than the cavity line width of $\kappa / 2 \pi \sim 7 \mathrm{kHz}$ [9]. This will allow to perform magnetic 


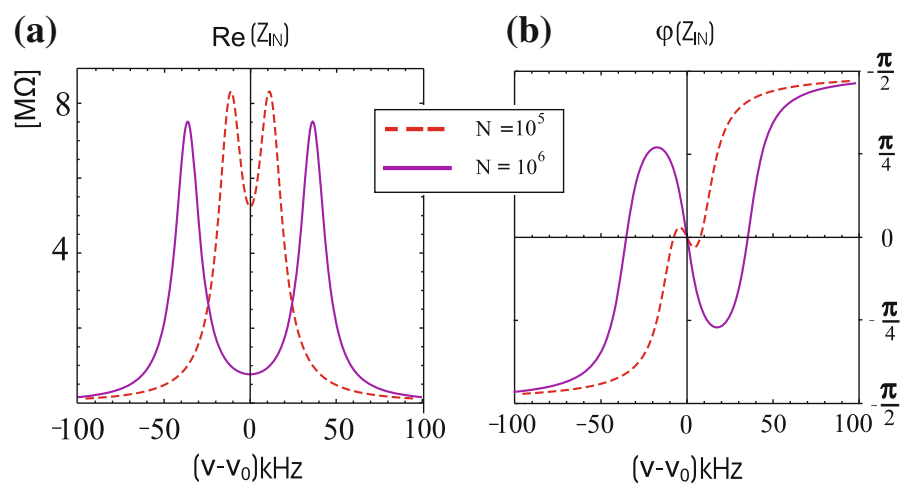

Fig. 7 Response of the atom-cavity system as illustrated by its complex impedance Z: a the real part of Z is related to the spectrum and $\mathbf{b}$ the phase of $Z$ directly illustrates the phase shift of the transmitted microwave radiation. The calculations are for $\mathrm{Q}=10^{6}, \mathrm{~N}=10^{6}$ and $\mathrm{N}=10^{5}$ and plotted versus incident field detuning from the resonance frequency

coupling cavity QED experiments with MW photons and collective state qubits stored in the atomic ensemble (see Fig. 7).

The most direct approach to state transfer from the SC qubit to the atomic ensemble would be to prepare all the atoms in state $|g\rangle$, the cavity field in vacuum $\left|0_{c}\right\rangle$, and choose the frequencies of the cavity mode and the atomic hyperfine transition to be the same, $\delta_{a c}=0$. Then, by tuning the SC qubit frequency to resonance with the cavity, $\delta_{q c}=0$, during time $\tau_{q c}$ such that $2 \eta_{q c} \tau_{q c}=\pi$, an arbitrary quantum state $|\psi\rangle=\alpha|0\rangle+\beta|1\rangle$ will be transferred from the SC qubit to the CPW cavity field (cf. Eq. 1). Next, it follows from Eq. (19) that the collective coupling rate of the cavity field and the atomic ensemble via the transition $\left|n_{g}=N, n_{s}=0 ; 1_{c}\right\rangle \rightarrow$ $\left|n_{g}=N-1, n_{s}=1 ; 0_{c}\right\rangle$ is given by $\sqrt{N} \eta_{a c} \sim 2 \pi \times 40 \mathrm{KHz}$. Thus, during time $\tau_{s g}=\pi /\left(2 \sqrt{N} \eta_{a c}\right) \sim 6 \mu \mathrm{s}$, corresponding to half a Rabi cycle, the cavity photon will be absorbed by the atoms and we will have achieved our goal. The time $\tau_{s g}$ is, however, comparable to the photon lifetime in the CPW cavity, $\kappa^{-1}=Q / \omega_{c} \sim 22 \mu \mathrm{s}$. Thus the photon will be lost with probability $P_{\text {loss }} \approx \kappa \tau_{s g} \sim 0.27$ before being coherently absorbed by the atoms. It is therefore necessary to improve the CPW cavity by increasing its quality factor $Q$ and thereby decreasing the photon decay rate $\kappa$.

In an alternative setup, the SC qubit and the atoms are tuned to be resonant with each other, $\delta_{q c, a c} \simeq \delta$, but detuned from the cavity mode frequency. For large detuning $\delta \gg \eta_{q c}$, the adiabatic elimination of the cavity mode yields an effective photon decay rate $\kappa_{\text {eff }}=\kappa \eta_{q c}^{2} / \delta^{2}$, while the corresponding second-order interaction Hamiltonian, $V_{q a}^{(2)}=\hbar \eta_{\mathrm{eff}}\left(\hat{s}^{\dagger} \hat{g} \hat{\sigma}^{-}+\hat{\sigma}^{+} \hat{g}^{\dagger} \hat{s}\right)$, with $\eta_{\mathrm{eff}}=\eta_{q c} \eta_{a c} / \delta$, describes an effective swap of an excitation between the SC qubit and atomic ensemble with the rate $\sqrt{N} \eta_{\text {eff }}$, mediated by virtual photon exchange in the cavity. Thus the effective coupling is reduced by a factor of $\delta / \eta_{q c}$, while the decoherence rate caused by the cavity is reduced by a factor of $\left(\delta / \eta_{q c}\right)^{2}$. For $\delta=10 \eta_{q c}$ we then have $\sqrt{N} \eta_{\text {eff }} \sim 2 \pi \times 4 \mathrm{KHz}$ while $\kappa_{\text {eff }} \simeq 2 \pi \times 100 \mathrm{~Hz}$, which yields a low probability of photon decay during the excitation swap, $P_{\text {loss }} \approx \kappa_{\text {eff }} \pi /\left(2 \sqrt{N} \eta_{\text {eff }}\right)=0.04$. 
Recall, however, that the SC qubit decoherence (dephasing) $\gamma_{q} \lesssim 1 \mathrm{MHz}$ is much larger than $\sqrt{N} \eta_{\mathrm{eff}}$, hampering the detuned scheme above. In turn, $\gamma_{q}$ is much smaller than the coupling $\eta_{q c}$, hence this decoherence does not pose a problem during the resonant excitation exchange between the SC qubit and CPW cavity. This rapid stage may therefore be accomplished with high fidelity, as opposed to the much slower stage of excitation transfer from the cavity to the atoms with weak magnetic dipole transition.

The dynamical control by modulation discussed in Sect. 3 can be used to suppress the SC qubit decoherence effects. It should be possible to improve both off- and on-resonant transfer fidelity, i.e., reduce the error by $50 \%$ (Fig. 4).

\subsection{Implementation in a NV center ensemble}

Magnetic coupling cavity QED can also be implemented using an ensemble of electron spins in diamond [36-38]. The ground $|g\rangle$, excited $|e\rangle$ and auxiliary $|x\rangle$ states of the NV color center in diamond correspond, respectively, to the $m=0, m=-1$ and $m=1$ Zeeman sublevels of the ground electronic (spin-triplet) state of the NV. The $|g\rangle \rightarrow|e\rangle,|x\rangle$ transition frequencies are around $2.88 \mathrm{GHz}$. The quantization axis of the electron spin orientation is given by the orientation in the crystal. A static magnetic field does not (to first order) change the orientation but can be used to tune the transition $|g\rangle \rightarrow|e\rangle$ in and out of resonance with the CPWR mode (see Fig. 8c). With a magnetic moment of $2 \mu_{B}$ the resulting coupling is similar to the coupling of the $\mathrm{Rb}$ ground state spins, but the ensemble shows strong inhomogeneous broadening of a few MHz.

In a typical experimental setup, Fig. 8a [38], a diamond is glued or pressed on top of the SC resonator chip. By placing the diamond sample at the center of the $\lambda / 2$

(a)

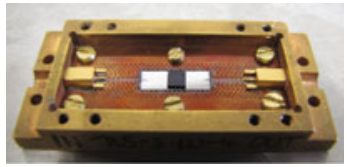

(c)

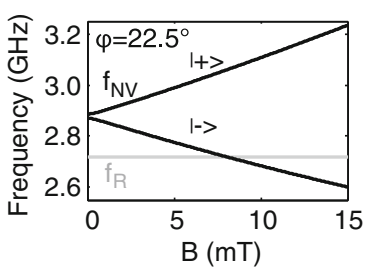

(b)

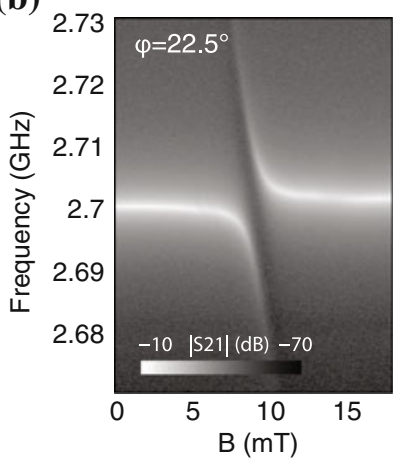

Fig. 8 a A (001) diamond sample with a high NV center density is placed on top of a SC-CPW resonator where the oscillating magnetic field possesses an anti-node. b NV center transition frequencies $|g\rangle \rightarrow|-1\rangle(|g\rangle \rightarrow|+1\rangle)$ as a function of an externally applied magnetic field. Since the resonance frequency of the CPWR is not changed by a magnetic field in the plane of the resonator, a static magnetic field can be used to tune the NV ensemble into resonance with the cavity. c Measured MW transmission $\left|S_{21}\right|^{2}$ versus applied magnetic field. A large Rabi splitting of $2 \times 8.7 \mathrm{MHz}$ is observed when ensemble and resonator are brought into resonance, an indication for strong magnetic coupling 
cavity one ensures that a large fraction of the magnetic mode volume is occupied by spins. A high concentration of NV centers can for example be obtained by irradiating a high-temperature high-pressure diamond of an initial nitrogen impurity concentration $\gtrsim 100 \mathrm{ppm}$ with neutrons and finally annealing the sample at $900{ }^{\circ} \mathrm{C}$.

An estimation of the coupling strength for $N \approx 10^{12} \mathrm{NV}$ center spins uniformly coupled to the same electromagnetic field mode results in $\sqrt{N} \eta_{a c} / 2 \pi \sim 10 \mathrm{MHz}$. Figure $8 \mathrm{~b}$ shows the experimentally observed avoided crossing originating from the magnetic spin-cavity coupling. In this measurement the cavity transmission is probed with a vector network analyzer while an external magnetic field is used to tune the spin ensemble. When both, NV center spins and cavity, have the same frequency, a large Rabi splitting of $2 \times 8.7 \mathrm{MHz}$ is observed.

Apart from coupling to electron spin ensembles, the hyperfine interaction between the NV centers electron spin and a nuclear spin in a neighboring lattice site can mediate the coupling to a nuclear spin ensemble. A demonstration of the coupling to ${ }^{13} \mathrm{C}$ nuclear spins in diamond with $I=\frac{1}{2}$ was shown in [38]. Although strong coupling was not yet observed, this is a first step towards a nuclear spin memory.

We will now illustrate the results of the optimized filtering discussed in Sect. 4 for the case of the in-homogeneously broadened NV ensembles. The degeneracy of levels $|e\rangle$ and $|x\rangle$ are lifted by stress in the crystal. The transitions $|g\rangle \rightarrow|e\rangle,|x\rangle$ can be selectively addressed by the external $\sigma_{ \pm}$-polarized microwave fields. We assume the inhomogeneous spectrum of the ensemble of NV centers has the total width of $\Delta / 2 \pi \sim 7 \mathrm{MHz}$, composed of three partially overlapping Lorentzians of widths $\sim 2.6 \mathrm{MHz}$ split by $\sim 2.2 \mathrm{MHz}$ due to the hyperfine coupling to the $I=1$ nuclear spin of the ${ }^{14} \mathrm{~N}$ atom.

For an ensemble-cavity coupling strength $\sqrt{N \overline{\eta^{2}}} \simeq 2 \pi \times 13 \mathrm{MHz}$, the excitation transfer time is $t_{\text {tr }}=\pi /\left(\sqrt{N \eta^{2}}\right) \approx 40 \mathrm{~ns}$. As an example, assume that one can achieve a CPWR with a quality factor of $Q=10^{6}$. The photon lifetime in the cavity, $\kappa^{-1} \approx 55 \mu \mathrm{s}$, is much longer than the transfer time. This allows a preparation that reduces the ensemble spectral width $\Delta$, and the number of active atoms $N$, by $5 \cdot 10^{3}$, almost 4 orders of magnitude, while still keeping the transfer time $t_{\mathrm{tr}} \approx 2.8 \mu \mathrm{s}=0.05 \kappa^{-1} \ll \kappa^{-1}$, well within the cavity lifetime. The new ensemble, created by the optimal preparation pulse $\Omega(t)$ of $T=0.7 \mathrm{~ms}$ duration, has the storage fidelity $\mathcal{F}(t) \approx 1-(t / 0.22 \mathrm{~ms})^{2}$. This ensemble hardly loses any fidelity during the transfer. Furthermore, it allows us to store QI for $t \simeq 50 \mu$ s with $95 \%$ fidelity, compared to 3 ns storage with the original ensemble and $2.8 \mu$ s storage in the cavity. Hence, we can outperform cavity storage even when $Q$ is high $\left(10^{6}\right)$.

By contrast, had we used the square preparation pulse, the new ensemble would have had a storage fidelity of $\mathcal{F}(t) \approx 1-t / 0.17 \mathrm{~ms}$, which can store QI for $t \simeq 8.5 \mu \mathrm{s}$ with $95 \%$ fidelity - more than 5 times worse than with the optimal preparation.

\section{Atom-cavity electric-dipole coupling via Rydberg states}

Very strong atom-cavity field coupling can be achieved at microwave frequencies for electric-dipole transitions between highly-excited Rydberg states [26,27]. This allows 


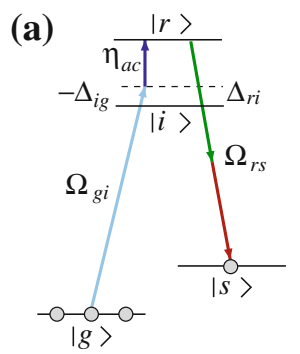

(b)

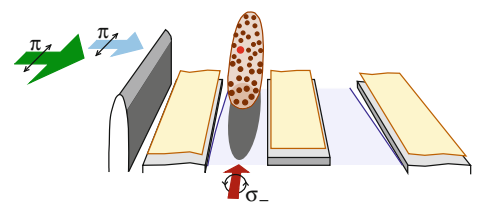

Fig. 9 a Atomic Rydberg states $|i\rangle$ and $|r\rangle$ and relevant couplings for the excitation transfer from the CPW cavity to the atomic storage state $|s\rangle$. b Propagation geometry of the corresponding optical fields and the beam-stop and metallic mirrors on top of the SC electrodes of the CPW cavity

the atoms to be placed much further away from the chip surface, and still achieve strong collective coupling. We would like to note here that we use the Rydberg states to mediate the coupling between the MW photons and the stored collective spin state of the atomic ensemble. Since the qubits give single MW photons at a time, we do not have to employ Rydberg blockade [82] to ensure the writing of single quantum states.

Let us select a pair of Rydberg states $|i\rangle$ and $|r\rangle$ such that the frequency $\omega_{r i}$ of transition $|i\rangle \leftrightarrow|r\rangle$ is close to the cavity mode frequency $\omega_{c}$, the corresponding detuning being $\delta_{r i}=\omega_{r i}-\omega_{c}$. We envision a level scheme sketched in Fig. 9(a), where the transition $|g\rangle \leftrightarrow|i\rangle$ is driven by an external optical field with Rabi frequency $\Omega_{g i}$ and detuning $\delta_{i g}$. The Hamiltonian reads

$$
\begin{aligned}
H_{a c}= & \hbar \delta_{i g} \hat{i}^{\dagger} \hat{i}+\hbar\left(\delta_{i g}+\delta_{r i}\right) \hat{r}^{\dagger} \hat{r} \\
& -\hbar\left(\Omega_{g i} \hat{i}^{\dagger} \hat{g}+\eta_{a c} \hat{r}^{\dagger} \hat{i} \hat{c}+\text { H.c. }\right),
\end{aligned}
$$

where $\eta_{a c}=\left(\wp_{i r} / \hbar\right) \varepsilon_{c} u(\mathbf{r})$ is the atom-cavity field coupling rate, $\wp_{i r}$ being the corresponding dipole matrix element, while operators $\hat{i}\left(\hat{i}^{\dagger}\right)$ and $\hat{r}\left(\hat{r}^{\dagger}\right)$ annihilate (create) an atom in state $|i\rangle$ and $|r\rangle$, respectively.

We set the detunings as $\delta_{r i} \simeq-\delta_{i g}=\delta$. Then, given a photon in the cavity, the external field $\Omega_{g i}$ and the cavity field induce a two-photon transition from the ground state $|g\rangle$ to the Rydberg state $|r\rangle$ via non-resonant intermediate Rydberg state $|i\rangle$. If $\delta \gg \eta_{a c}, \sqrt{N} \Omega_{g i}$, state $|i\rangle$ is never populated, and we obtain an effective interaction Hamiltonian $V_{a c}^{(2)}=\hbar \eta_{\text {eff }}\left(\hat{r}^{\dagger} \hat{g} \hat{c}+\hat{c}^{\dagger} \hat{g}^{\dagger} \hat{r}\right)$, with $\eta_{\text {eff }}=\Omega_{g i} \eta_{a c} / \delta$. Thus, starting from the initial state of the system $\left|n_{g}=N, n_{i, r, s}=0 ; 1_{c}\right\rangle$, by pulsing $\Omega_{g i}$ for time $\tau_{g r}=\pi /\left(2 \sqrt{N} \eta_{\text {eff }}\right)$, the cavity photon will be coherently absorbed and a single atom from the ensemble will be excited to the Rydberg state $|r\rangle$. Next, another (bichromatic) external field with Rabi frequency $\Omega_{r s}$ pulsed for a time $\tau_{r s}=\pi /\left(2 \Omega_{r s}\right)$ can resonantly transfer the single collective Rydberg excitation of the atomic ensemble to the storage state $|s\rangle$ : this process is described by $H_{r s}=-\hbar \Omega_{r s} \hat{s}^{\dagger} \hat{r}+$ H.c. At a later time, when required, the reverse process can add a single photon in the cavity while all the atoms will end up in state $|g\rangle$. This single photonic excitation can then be quickly transferred to the SC qubit, as described above. 
In what follows, we survey the relevant experimental parameters. An elongated trapping volume $V_{a} \sim d \times d \times l$, with $d \simeq 5 \mu \mathrm{m}$ and $l \simeq 1 \mathrm{~mm}$, contains $N \simeq 10^{6}$ atoms at density $\rho_{a} \sim 4 \times 10^{13} \mathrm{~cm}^{-3}$. The atomic lower states $|g\rangle$ and $|s\rangle$ correspond to the $\left|F=1, M_{F}=-1\right\rangle$ and $\left|F=2, M_{F}=1\right\rangle$ sublevels of the ground electronic state $5 s_{1 / 2}$ of ${ }^{87} \mathrm{Rb}$. We choose the Rydberg states $|i\rangle \equiv\left|n p_{1 / 2}, F=2, M_{F}=-1\right\rangle$ and $|r\rangle \equiv\left|(n+1) s_{1 / 2}, F=1, M_{F}=0\right\rangle$ with $n=68$ the principal quantum number. The quantum defects for the $s_{1 / 2}$ and $p_{1 / 2}$ Rydberg states of Rb are $\delta_{s}=3.131$ and $\delta_{p}=2.6545$ [26], with which the corresponding transition frequency is $\omega_{r i}=$ $2 \pi \times 12.2 \mathrm{GHz}$. Calculation of the relevant transition dipole matrix element involving the radial and angular parts gives $\wp_{i r} \simeq 1520 a_{0} e$.

With the strip-line length $L \simeq 1 \mathrm{~cm}$ and effective dielectric constant $\epsilon_{r} \sim 6$, the frequency of the $m$ th standing-wave mode of the cavity is $\omega_{c}=\pi m c / L \sqrt{\epsilon_{r}}$ (Fig. 1a). The grounded SC electrodes at distance $w \simeq 10 \mu \mathrm{m}$ confine the cavity field within the effective volume $V_{c}=\int d^{3} r|u(\mathbf{r})|^{2} \simeq \frac{\pi}{2} w^{2} L$ yielding $\varepsilon_{c}=\sqrt{\hbar \omega_{c} / 2 \epsilon_{0} V_{c}} \gtrsim$ $0.5 \mathrm{~V} / \mathrm{m}$. Taking the full-wavelength $(m=2)$ linearly polarized cavity mode with $\omega_{c} / 2 \pi \simeq 12.16 \mathrm{GHz}$, we estimate [9] that at the position of atomic cloud about $10 \mu \mathrm{m}$ above the CPW surface the mode function $u(\mathbf{r}) \simeq e^{-1}$ which yields the vacuum Rabi frequency $\eta_{a c}=\left(\wp_{i r} / \hbar\right) \varepsilon_{c} u(\mathbf{r}) \simeq 2 \pi \times 3.85 \mathrm{MHz}$ and appropriately large detuning $\delta_{r i} \simeq 10 \eta_{a c}$.

The transition $|g\rangle \rightarrow|i\rangle$ is driven by linearly $\pi$-polarized UV field with wavelength $\lambda_{i g} \simeq 297 \mathrm{~nm}$ and detuning $\delta_{i g}=-\delta_{r i}$. To optimize the transition rate, its Rabi frequency is chosen as $\sqrt{N} \Omega_{g i} \simeq \eta_{a c}$, with which the transfer time is $\tau_{g r} \simeq 0.65 \mu \mathrm{s}$. The required UV field intensity at the atomic cloud is $I_{g i}=0.46 \mathrm{~W} \mathrm{~cm}^{-2}$. Next, $|r\rangle \rightarrow|s\rangle$ is a two-photon transition via non-resonant intermediate state $\mid 5 p_{1 / 2}, F=$ $\left.2, M_{F}=0\right\rangle=|e\rangle$. The wavelengths are $\lambda_{r e} \simeq 474 \mathrm{~nm}$ (linearly $\pi$-polarized field) and $\lambda_{e s} \simeq 795 \mathrm{~nm}$ (circularly $\sigma_{-}$-polarized field). With the corresponding intensities $I_{r e}=440 \mathrm{~W} \mathrm{~cm}^{-2}$ and $I_{e s}=2.25 \mathrm{~mW} \mathrm{~cm}^{-2}$ and intermediate detuning $\delta_{e s}=$ $2 \pi \times 25 \mathrm{MHz}$, the two-photon Rabi frequency is $\Omega_{r s}=2 \pi \times 250 \mathrm{KHz}$ leading to the transfer time of $\tau_{r s} \simeq 1 \mu \mathrm{s}$. Note that $\tau_{g r}$ and $\tau_{r s}$ are short compared to the lifetimes of cavity photon $1 / \kappa \sim 10 \mu$ s and Rydberg states $1 / \gamma_{R} \sim 100 \mu$ s [26].

Figure 10 shows the results of numerical integration of the master equation $[83,84]$ for density operator $\hat{\rho}(t)$ whose evolution is governed by Hamiltonians $H_{q c}, H_{a c}$ of (20) and $H_{r s}$ with the addition of the cavity decay and SC qubit dephasing times as discussed in Sect. 2. As seen, in the case of $\left\langle\hat{n}_{c}(0)\right\rangle=0$, the state transfer is nearly ideal, with the small final error probability $P_{\text {err }} \lesssim 0.04$ due to relaxation of the qubit, the cavity field and the atoms. However, in the case of finite temperature, $\left\langle\hat{n}_{c}(0)\right\rangle=0.5$, during the transfer, as expected, the cavity field and the collective atomic state occupation numbers $\left\langle\hat{n}_{c}\right\rangle$ and $\left\langle\hat{n}_{r, s}\right\rangle$ exceed unity, and the resulting error probability $P_{\text {err }} \simeq 0.3$ is large. Hence, suppressing the cavity temperature is crucial for achieving high transfer fidelity.

We have perfomed simulations of the dynamics of the SC qubit-cavity transfer process with temperature-dependent initial population $\left\langle\hat{n}_{c}(0)\right\rangle$ of the CPW cavity photon field, for typical frequencies of few $\mathrm{GHz}[4,25,48-50]$. We characterize the transfer process for a given initial state $|\psi\rangle_{q}$ of the SC qubit by the conditional fidelity $F_{\psi}=\operatorname{Tr}\left(\hat{\rho}\left|\psi_{f}\right\rangle\left\langle\psi_{f}\right|\right)$, where $\left|\psi_{f}\right\rangle$ denotes the final state stored in the memory for 


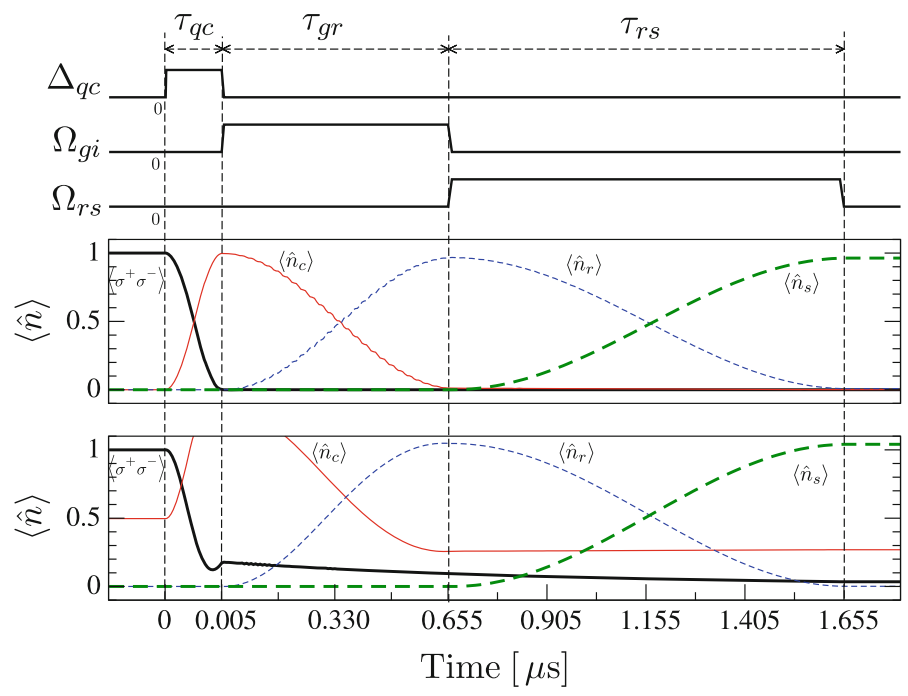

Fig. 10 Numerical simulations of the three-step excitation transfer from the SC qubit to the ultracold atomic ensemble. The cavity is taken to have a $Q$-factor of $10^{6}$ and the SC qubit is taken to have a dephasing time of $1 / \gamma_{q} \gtrsim 1 \mu$ s. Top panel illustrates the sequence of $(\pi-)$ pulses: first the SQ qubit is brought to resonance with the CPW cavity, by pulsing $\delta_{q c}(t)=0$ for time $\tau_{q c}$; next, the $\Omega_{g i}(t)$ field is pulsed for time $\tau_{g r}$; finally, the $\Omega_{r s}(t)$ field is pulsed for time $\tau_{r s}$. Central and lower panels show the dynamics of occupation numbers for the qubit exited state $\left\langle\hat{\sigma}^{+} \hat{\sigma}^{-}\right\rangle$, the cavity field $\left\langle\hat{n}_{c}\right\rangle \equiv\left\langle\hat{c}^{\dagger} \hat{c}\right\rangle$ and the collective atomic states $\left\langle\hat{n}_{r}\right\rangle \equiv\left\langle\hat{r}^{\dagger} \hat{r}\right\rangle$ and $\left\langle\hat{n}_{s}\right\rangle \equiv\left\langle\hat{s}^{\dagger} \hat{s}\right\rangle$. Initially, the mean thermal photon number is $\left\langle\hat{n}_{c}(0)\right\rangle=0$ in the central panel, and $\left\langle\hat{n}_{c}(0)\right\rangle=0.5$ in the lower panel

an ideal transfer. The mean transfer fidelity $\bar{F}$ is obtained by averaging $F_{\psi}$ over all possible $|\psi\rangle$. The dependence of $F_{\psi}$ and $\bar{F}$ on the CPW cavity temperature $T$, or the mean thermal photon number $\left\langle\hat{n}_{c}\right\rangle=\left(e^{\hbar \omega_{c} / k_{\mathrm{B}} T}-1\right)^{-1}$, is shown in Fig. 11. Below $k_{\mathrm{B}} T / \hbar \omega_{c} \simeq 0.2$, corresponding to $\left\langle\hat{n}_{c}\right\rangle \lesssim 0.01$, the transfer fidelity is fairly high, $\bar{F}>98 \%$, but then it quickly degrades due to the detrimental effect of even a small number of thermal photons. For the above parameters this critical temperature is $T \sim 0.1 \mathrm{~K}$ necessitating cryogenic conditions. Hence the importance of keeping the cavity temperature very low (see Sect. 2).

\section{Conclusions}

We have discussed here two general strategies for quantum state-transfer and storage optimization within hybrid open systems, and their consequences for experimental implementation on a SC chip.

A: Our analysis of dynamically controlled transfer from a "noisy" qubit to its "quiet" counterpart, has revealed an intriguing interplay between the ability to avoid bathinduced errors that profoundly depend on the bath-memory time and the limitations imposed by leakage out of the operational subspace. Counterintuitively, under no circumstances is the fastest transfer optimal (for a given transfer energy).

An important issue that merits discussion in this context is that of cavity relaxation effects. The situation studied here pertains to qubits where the main source of decoherence is dephasing noise. Yet, since the noise suppression attained here 


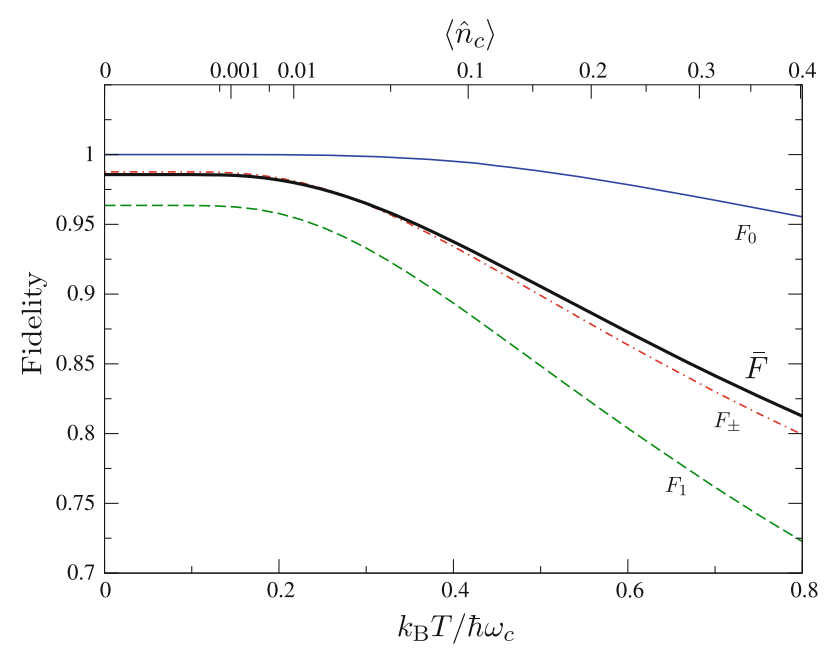

Fig. 11 Conditional $F_{\psi}$ and mean $\bar{F}$ transfer fidelities vs temperature $k_{\mathrm{B}} T$ (lower horizontal axis) or mean thermal photon number $\left\langle\hat{n}_{c}\right\rangle$ (upper horizontal axis). In $F_{\psi}, \psi=0,1, \pm$ correspond to states $|0\rangle,|1\rangle$ and $\frac{1}{\sqrt{2}}[|0\rangle \pm(i)|1\rangle]$

requires longer transfer time than that of the fastest transfer, one may wonder whether the (competing) cavity relaxation effects may not increase as well. However, although the total transfer time is increased, the main part of the transfer takes actually less time than in the fastest case. Hence, a reduction in cavity relaxation may occur, similar to the dephasing reduction: this is indeed confirmed by our simulation results.

$B$ : We have proposed a method that alleviates the shortcomings of the spin-ensemble inhomogeneous broadening, assuming that the cavity lifetime is much longer than the transfer time. This method allows us to optimize the memory, given the cavity, the spectral density of the ensemble and their mean coupling strength. This protocol is compatible with dynamical control [61-65], especially with its optimal version $[54,55,85]$ which can help mitigate the qubit decoherence, while the cavity temperature effects can be partially tolerated [6,28] or suppressed [86]. When the spin-ensemble temperature is an issue, dynamical initialization may be useful [87-89].

Three possible experimental implementations of the above methods have been discussed: (i) magnetic dipole coupling of CPWR to ultracold atom ensembles; (ii) magnetic dipole coupling of CPWR to NV-center ensembles; (iii) electric dipole coupling to Rydberg atom ensembles.

Overall, we have developed a promising approach for realizing efficient quantum state transfer between superconducting charge qubits and mesoscopic atomic or spin ensembles coupled to a microwave coplanar waveguide cavity. Our estimates show that this hybrid system may allow longer and higher-fidelity storage than even a high-Q cavity.

Acknowledgments This research was supported by the EU through MIDAS, by DIP, the Humboldt-Meitner Award (G.K.), the Humboldt Foundation (D.P.) and the Wittgenstein Prize (J.S.). 
Open Access This article is distributed under the terms of the Creative Commons Attribution Noncommercial License which permits any noncommercial use, distribution, and reproduction in any medium, provided the original author(s) and source are credited.

\section{References}

1. Andre, A. et al.: Polar molecules near superconducting resonators: a coherent, all-electrical atom-mesocopic interface. Nat. Phys. 2, 636 (2006)

2. Rabl, P. et al.: Hybrid quantum processors: molecular ensembles as quantum memory for solid state circuits. Phys. Rev. Lett. 97(3), 033003 (2006)

3. Tordrup, Karl, Mølmer, Klaus: Quantum computing with a single molecular ensemble and a cooper-pair box. Phys. Rev. A 77(2), 020301 (2008)

4. Schoelkopf, R.J., Girvin, S.M.: Wiring up quantum systems. Nature 451, 664 (2008)

5. Petrosyan, D., Fleischhauer, M.: Quantum information processing with single photons and atomic ensembles in microwave coplanar waveguide resonators. Phys. Rev. Lett. 100, 170501 (2008)

6. Petrosyan, D. et al.: Reversible state transfer between superconducting qubits and atomic ensembles. Phys. Rev. A 79(4), 040304(R) (2009)

7. Wallquist, M. et al.: Hybrid quantum devices and quantum engineering. Phys. Scr. t137, 014001 (2009)

8. Imamoglu, A.: Cavity qed based on collective magnetic dipole coupling: spin ensembles as hybrid two-level systems. Phys. Rev. Lett. 102(8), 083602 (2009)

9. Verdú, J. et al.: Strong magnetic coupling of an ultracold gas to a superconducting waveguide cavity. Phys. Rev. Lett. 103(4), 043603 (2009)

10. Devoret, M.H., Martinis, J.M.: Implementing qubits with superconducting integrated circuits. Quantum Inf. Process. 3, 163-203 (2004)

11. You, J.Q., Nori, F.: Superconducting circuits and quantum information. Phys. Today 58(11), 4247 (2005)

12. Makhlin, Y., Schön, G., Shnirman, A.: Quantum-state engineering with Josephson-junction devices. Rev. Mod. Phys. 73(2), 357-400 (2001)

13. You, J.Q., Nori, F.: Atomic physics and quantum optics using superconducting circuits. Nature 474, 589 (2011)

14. Duan, L.-M., Monroe, C.: Colloquium: quantum networks with trapped ions. Rev. Mod. Phys. 82(2), 1209-1224 (2010)

15. Taylor, J.M., Marcus, C.M., Lukin, M.D.: Long-lived memory for mesoscopic quantum bits. Phys. Rev. Lett. 90(20), 206803 (2003)

16. Fleischhauer, M., Lukin, M.D.: Quantum memory for photons: dark-state polaritons. Phys. Rev. A 65(2), 022314 (2002)

17. Lukin, M.D.: Colloquium: trapping and manipulating photon states in atomic ensembles. Rev. Mod. Phys. 75(2), 457-472 (2003)

18. Zhao, B. et al.: A millisecond quantum memory for scalable quantum networks. Nat. Phys. 5, 95 (2009)

19. Dudin, Y.O., Zhao, R., Kennedy, T.A.B., Kuzmich, A.: Light storage in a magnetically dressed optical lattice. Phys. Rev. A 81(4), 041805 (2010)

20. Deutsch, C. et al.: Spin self-rephasing and very long coherence times in a trapped atomic ensemble. Phys. Rev. Lett. 105(2), 020401 (2010)

21. Gurudev Dutt, M.V. et al.: Quantum register based on individual electronic and nuclear spin qubits in diamond. Science 316(5829), 1312-1316 (2007)

22. Taylor, J.M. et al.: High-sensitivity diamond magnetometer with nanoscale resolution. Nat. Phys. 4, 810-816 (2008)

23. Acosta, V.M. et al.: Diamonds with a high density of nitrogen-vacancy centers for magnetometry applications. Phys. Rev. B 80(11), 115202 (2009)

24. Stanwix, P.L. et al.: Coherence of nitrogen-vacancy electronic spin ensembles in diamond. Phys. Rev. B 82(20), 201201 (2010)

25. Blais, A. et al.: Cavity quantum electrodynamics for superconducting electrical circuits: an architecture for quantum computation. Phys. Rev. A 69(6), 062320 (2004)

26. Gallagher, T.F.: Rydberg Atoms. Cambridge University Press, Cambridge (1994)

27. Brune, M., Raimond, J.-M., Haroche, S.: Manipulating quantum entanglement with atoms and photons in a cavity. Rev. Mod. Phys. 73, 565 (2001) 
28. Henschel, K., Majer, J., Schmiedmayer, J., Ritsch, H.: Cavity qed with an ultracold ensemble on a chip: prospects for strong magnetic coupling at finite temperatures. Phys. Rev. A 82(3), 033810 (2010)

29. Nirrengarten, T. et al.: Realization of a superconducting atom chip. Phys. Rev. Lett. 97(20), 200405 (2006)

30. Mukai, T. et al.: Persistent supercurrent atom chip. Phys. Rev. Lett. 98(26), 260407 (2007)

31. Roux, C. et al.: Bose-Einstein condensation on a superconducting atom chip. Europhys. Lett. 81, 56004 (2008)

32. Kasch, B. et al.: Cold atoms near superconductors: atomic spin coherence beyond the Johnson noise limit. New J. Phys. 12, 065024 (2010)

33. Hufnagel, Ch., Mukai, T., Shimizu, F.: Stability of a superconductive atom chip with presistent current. Phys. Rev. A 79, 053641 (2009)

34. Emmert, A. et al.: Measurement of the trapping lifetime close to a cold metallic surface on a cryogenic atom-chip. Eur. Phys. J. D 51, 173 (2009)

35. Meek, S.A., Conrad, H., Meijer, G.: Trapping molecules on a chip. Science 324, 1699-1702 (2009)

36. Schuster, D.I. et al.: High-cooperativity coupling of electron-spin ensembles to superconducting cavities. Phys. Rev. Lett. 105(14), 140501 (2010)

37. Kubo, Y. et al.: Strong coupling of a spin ensemble to a superconducting resonator. Phys. Rev. Lett. 105(14), 140502 (2010)

38. Amsüss, R. et al:: Cavity qed with magnetically coupled collective spin states. Phys. Rev. Lett. 107(6), 060502 (2011)

39. Wu, H. et al.: Storage of multiple coherent microwave excitations in an electron spin ensemble. Phys. Rev. Lett. 105(14), 140503 (2010)

40. Bushev, P. et al.: Ultralow-power spectroscopy of a rare-earth spin ensemble using a superconducting resonator. Phys. Rev. B 84(6), 060501 (2011)

41. Reichel, J., Vuletic, V. (eds.): Atom Chips. Wiley, VCH (2011)

42. Roos, I., Mølmer, K.: Quantum computing with an inhomogeneously broadened ensemble of ions: Suppression of errors from detuning variations by specially adapted pulses and coherent population trapping. Phys. Rev. A 69(2), 022321 (2004)

43. Tordrup, K., Mølmer, K.: Quantum-state reconstruction with imperfect rotations on an inhomogeneously broadened ensemble of qubits. Phys. Rev. A 75(4), 042318 (2007)

44. Wesenberg, J.H., Kurucz, Z., Mølmer, K.: Dynamics of the collective modes of an inhomogeneous spin ensemble in a cavity. Phys. Rev. A 83(2), 023826 (2011)

45. Hahn, E.L.: Spin echoes. Phys. Rev. 80(8), 580-594 (1950)

46. Nilsson, M., Kröll, S.: Solid state quantum memory using complete absorption and re-emission of photons by tailored and externally controlled inhomogeneous absorption profiles. Opt. Commun. 247(4-6), 393-403 (2005)

47. Alexander, A.L., Longdell, J.J., Sellars, M.J., Manson, N.B.: Photon echoes produced by switching electric fields. Phys. Rev. Lett. 96(4), 043602 (2006)

48. Wallraff, A. et al.: Strong coupling of a single photon to a superconducting qubit using circuit quantum electrodynamics. Nature 431, 162-167 (2004)

49. Majer, J. et al.: Coupling superconducting qubits via a cavity bus. Nature 449, 443-447 (2007)

50. Sillanpaa, M.A., Park, J.I., Simmonds, R.W.: Coherent quantum state storage and transfer between two phase qubits via a resonant cavity. Nature 449, 438-442 (2007)

51. Brennecke, F. et al.: Cavity qed with a Bose-Einstein condensate. Nature 450, 268-271 (2007)

52. Mariantoni, M. et al.: Photon shell game in three-resonator circuit quantum electrodynamics. Nat. Phys. 7, 287 (2011)

53. Houck, A.A. et al.: Controlling the spontaneous emission of a superconducting transmon qubit. Phys. Rev. Lett. 101, 080502 (2008)

54. Clausen, J., Bensky, G., Kurizki, G.: Bath-optimized minimal-energy protection of quantum operations from decoherence. Phys. Rev. Lett. 104(4), 040401 (2010)

55. Gordon, G., Kurizki, G., Lidar, D.A.: Optimal dynamical decoherence control of a qubit. Phys. Rev. Lett. 101(1), 010403 (2008)

56. Wu, L.A., Kurizki, G., Brumer, P.: Master equation and control of an open quantum system with leakage. Phys. Rev. Lett. 102, 080405 (2009)

57. Wu, L.-A., Byrd, M.S., Lidar, D.A.: Polynomial-time simulation of pairing models on a quantum computer. Phys. Rev. Lett. 89, 057904 (2002)

58. Byrd, M.S., Wu, L-A., Lidar, D.A.: Overview of quantum error prevention and leakage elimination. J. Mod. Opt. 51, 2449 (2004) 
59. Byrd, M.S., Lidar, D.A., Wu, L-A., Zanardi, P.: Universal leakage elimination. Phys. Rev. A 71, 052301 (2005)

60. Lidar, D.A.: Towards fault tolerant adiabatic quantum computation. Phys. Rev. Lett. 100, 160506(2008)

61. Kofman, A.G., Kurizki, G.: Unified theory of dynamically suppressed qubit decoherence in thermal baths. Phys. Rev. Lett. 93(13), 130406 (2004)

62. Kofman, A.G., Kurizki, G.: Universal dynamical control of quantum mechanical decay: Modulation of the coupling to the continuum. Phys. Rev. Lett. 87, 270405 (2001)

63. Kofman, A.G., Kurizki, G.: Acceleration of quantum decay processes by frequent observations. Nature (London) 405, 546 (2000)

64. Gordon, G., Erez, N., Kurizki, G.: Universal dynamical decoherence control of noisy single- and multi-qubit systems. J. Phys. B 40(9), S75 (2007)

65. Gordon, G., Kurizki, G.: Universal dephasing control during quantum computation. Phys. Rev. A 76(4), 042310 (2007)

66. Breuer, H.P., Petruccione, F.: The Theory of Open Quantum Systems. Oxford University Press, Oxford (2002)

67. Viola, L., Knill, E., Lloyd, S.: Dynamical generation of noiseless quantum subsystems. Phys. Rev. Lett. 85(16), 3520-3523 (2000)

68. Viola, L., Knill, E., Lloyd, S.: Dynamical decoupling of open quantum systems. Phys. Rev. Lett. 82(12), 2417-2421 (1999)

69. Viola, L., Lloyd, S.: Dynamical suppression of decoherence in two-state quantum systems. Phys. Rev. A 58(4), 2733-2744 (1998)

70. Vitali, D., Tombesi, P.: Heating and decoherence suppression using decoupling techniques. Phys. Rev. A 65(1), 012305 (2001)

71. Uhrig, G.S.: Keeping a quantum bit alive by optimized $\pi$-pulse sequences. Phys. Rev. Lett. 98(10), 100504 (2007)

72. Kofman, A.G., Kurizki, G.: Quantum Zeno effect on atomic excitation decay in resonators. Phys. Rev. A 54(5), R3750-R3753 (1996)

73. Abragam, A.: The Principles of Nuclear Magnetism. Oxford University Press, Oxford, England (1961)

74. Allen, L., Eberly, J.H.: Optical Resonance and Two-Level Atoms. Wiley, New York (1975)

75. Folman, R. et al.: Controlling cold atoms using nanofabricated surfaces: atom chips. Phys. Rev. Lett. 84, 4749 (2000)

76. Folman, R. et al.: Microscopic atom optics: from wires to an atom chip. Adv. At. Mol. Opt. Phys. 48, 263-356 (2002)

77. Fortagh, J., Zimmermann, C.: Magnetic microtraps for ultracold atoms. Rev. Mod. Phys. 79, 235 (2007)

78. Lin, Y., Teper, I., Chin, C., Vuletić, V.: Impact of the Casimir-Polder potential and Johnson noise on Bose-Einstein condensate stability near surfaces. Phys. Rev. Lett. 92, 50404 (2004)

79. Aigner, S. et al.: Long-range order in electronic transport through disordered metal films. Science 319, 1226-1229 (2008)

80. Haslinger, S. et al.: Electron beam driven alkali metal atom source for loading a magneto-optical trap in a cryogenic environment. Appl. Phys. B 102, 819 (2011)

81. Haslinger, S.: Cold atoms in a cryogenic environment. PhD thesis, TU-Wien (2011)

82. Lukin, M.D. et al.: Dipole blockade and quantum information processing in mesoscopic atomic ensembles. Phys. Rev. Lett. 87(3), 037901 (2001)

83. Nielsen, M., Chuang, I.: Quantum Computation and Quantum Information. Cambridge University Press, Cambridge (2000)

84. Lambropoulos, P., Petrosyan, D.: Fundamentals of Quantum Optics and Quantum Information. Springer, Berlin (2006)

85. Bensky, G. et al.: Universal dynamical decoupling from slow noise with minimal control. Europhys. Lett. 89(1), 10011 (2010)

86. Zhang, P., Wang, Y.D., Sun, C.P.: Cooling mechanism for a nanomechanical resonator by periodic coupling to a cooper pair box. Phys. Rev. Lett. 95(9), 097204 (2005)

87. Erez, N., Gordon, G., Nest, M., Kurizki, G.: Thermodynamic control by frequent quantum measurements. Nature 452, 724-727 (2008)

88. Gordon, G. et al.: Cooling down quantum bits on ultrashort time scales. New J. Phys. 11(12), 123025 (2009)

89. Álvarez Gonzalo, A., Bhaktavatsala Rao, D.D., Frydman, L., Kurizki, G.: Zeno and anti-Zeno polarization control of spin ensembles by induced dephasing. Phys. Rev. Lett. 105(16), 160401 (2010) 